\title{
Curcumin attenuates the development of thoracic aortic aneurysm by inhibiting VEGF expression and inflammation
}

\author{
XIANG LI $^{1}$, QIN FANG $^{2}$, XIAOHONG TIAN ${ }^{3}$, XIAOHONG WANG $^{3}$, \\ QIANG AO ${ }^{3}$, WEIJIAN HOU ${ }^{3}, \mathrm{HAO} \mathrm{TONG}^{3}, \mathrm{JUN} \mathrm{FAN}^{3}$ and SHULING BAI ${ }^{3}$ \\ ${ }^{1}$ Department of Cell Biology, China Medical University; ${ }^{2}$ Department of Cardiac Surgery, \\ First Hospital of China Medical University; ${ }^{3}$ Department of Tissue Engineering, School of \\ Fundamental Science, China Medical University, Shenyang, Liaoning 110122, P.R. China
}

Received August 19, 2016; Accepted May 9, 2017

DOI: $10.3892 / \mathrm{mmr} .2017 .7169$

\begin{abstract}
Angiogenesis is an important process in the pathogenesis of aortic aneurysm. The aim of the present study was to investigate the angiogenic balance and the expression of vascular endothelial growth factor (VEGF) in thoracic aortic aneurysm (TAA). A previous study demonstrated that curcumin exerts a marked effect on aortic aneurysm development. Therefore, the present study determined whether curcumin is able to modulate angiogenesis and inflammatory signaling in TAA by collecting human TAA samples and establishing a rat TAA model using periaortic application of $\mathrm{CaCl}_{2}$. TAA rats were treated with curcumin or $1 \%$ carboxymethyl cellulose and were sacrificed 4 weeks after the operation. All tissue specimens were analyzed by histological staining, immunohistochemistry and western blotting. Human TAA samples exhibited increased neovascularization and VEGF expression when compared with normal aortic walls. In rat tissues, treatment with curcumin resulted in reduced aneurysm size and restored the wavy structure of the elastic lamellae. In addition, curcumin decreased neovascularization and the expression of VEGF. Immunohistochemical analysis indicated that curcumin significantly inhibited infiltration of cluster of differentiation (CD) $3^{+}$and $\mathrm{CD} 68^{+}$cells in TAA. Furthermore, curcumin treatment decreased the expression of vascular cell adhesion molecule-1, intracellular adhesion molecule-1, monocyte chemoattractant protein-1 and tumor necrosis factor- $\alpha$. Collectively, the results demonstrated that angiogenesis and VEGF expression were increased in the aortic wall in TAA. Treatment with curcumin inhibited TAA development in rats, which was associated with suppression of VEGF expression.
\end{abstract}

Correspondence to: Dr Jun Fan, Department of Tissue Engineering, School of Fundamental Science, China Medical University, 77 Puhe Road, Shenyang North New Area, Shenyang, Liaoning 110122, P.R. China

E-mail: jfan@cmu.edu.cn

Key words: angiogenesis, aortic aneurysm, inflammation, curcumin, vascular endothelial growth factor, rat
In addition, curcumin attenuated inflammatory cell infiltration and suppressed inflammatory factor expression in the periaortic tissue of TAA.

\section{Introduction}

Thoracic aortic aneurysm (TAA) is often asymptomatic and is associated with a high degree of morbidity and mortality (1). The histopathological abnormalities underlying TAA include medial degeneration, loss of smooth muscle cells, damage to elastic fiber and infiltration of inflammatory cells in the aortic wall (2). However, the mechanism underlying the development of TAA is unclear. The conventional treatment for TAA is open chest surgical repair using prosthetic grafts. Therefore, the identification of novel methods that are open to modulation, and also pharmacotherapeutic drugs that limit or inhibit aortic aneurysm development, are required.

Increased adventitial vasculogenesis is one of the pathological features of abdominal aortic aneurysm (AAA) $(3,4)$. Angiogenesis in AAA has been associated with the risk of rupture and further complications (5). A previous study also indicated that in human TAA there is an increased density of microvessels originating from the adventitia when compared with healthy aortas (6). Angiogenesis is thought to serve an important role in the development of aortic aneurysms by creating a conduit for inflammatory cell transport and establishing chronic inflammation in the aortic wall (7). A strong association between vascular inflammation and the progression of aortic aneurysm has also been reported from clinical findings and animal experiments $(8,9)$. Vascular endothelial growth factor (VEGF) is known to be a significant regulator of angiogenesis and simultaneously stimulates elastolytic proteinases (10). VEGF can induce endothelial cell migration and proliferation, as well as vascular permeability $(10,11)$. In addition, it has been revealed that VEGF serves a crucial role in the development of AAA (12).

Curcumin is purified from the roots of the Curcuma longa plant and has been used to treat a number of diseases including cancer, osteoarthritis, diabetic microangiopathy and retinopathy, and inflammatory diseases $(13,14)$. An increasing body of evidence has demonstrated the therapeutic effects associated with curcumin, including anti-fibrotic and antioxidative 
activities, and cardioprotective effects, particularly in the treatment of cancer and anti-inflammatory diseases (15-17). It has been established that curcumin exerts anti-inflammatory effects and thus suppresses the development of experimental AAA in rats (18). In addition, curcumin may inhibit the c-Jun N-terminal kinase signaling pathway and apoptosis in rats with aortic aneurysms (19). Curcumin is known to be a novel antiangiogenic agent due to its effects on angiogenesis, which is associated with numerous pathological conditions including tumor progression and cardiovascular dysfunction (20-22). However, the effects of curcumin on angiogenesis and inflammation in TAA have yet to be elucidated.

The present study investigated angiogenesis and the expression of VEGF in TAA, as well as the protective potential of curcumin, with a particular focus on the modulation of VEGF expression and inflammation in TAA.

\section{Materials and methods}

Human aortic samples. Specimens were obtained from 12 patients who had undergone elective surgical repair of TAA

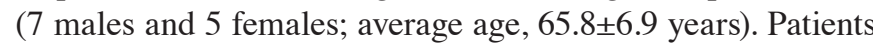
with degenerative aortic aneurysms recruited to the present study did not have connective tissue disorders, familial aortopathy or bicuspid aortic valve aortopathy, and aneurysms $>6 \mathrm{~cm}$ in diameter were selected. All patients were admitted to the China Medical University Hospital (Liaoning, China) between January 2009 and December 2014. Patients with recent infection, active inflammatory disorder, malignancy, collagen disease or chronic renal failure were not included in the study. Normal aorta samples (used as controls) were obtained from heart transplantation donors $(n=5)$. Controls were age-matched and asymptomatic, with no significant history of medication. The investigation conformed to the principles outlined in The Declaration of Helsinki. All protocols using human samples were approved by the Institutional Review Board of China Medical University Hospital and samples were obtained with written informed consent.

Animal use and surgical procedures. All animal experiments were conducted in strict accordance with the recommendations in the National Institutes of Health Guide for the Care and Use of Laboratory Animals (23). The protocol was approved by the Institutional Animal Care and Use Committee of China Medical University. A total of 30 male Wistar rats (weight, 200-250 g; age, 7-8 weeks; Experimental Animal Center of China Medical University, Liaoning, China) were used in the present study. The animals were housed in cages at room temperature $\left(25 \pm 1^{\circ} \mathrm{C}\right)$ and $50 \%$ humidity, with a 12 -h light/dark cycle and free access to chow and water. Rat TAA models were induced by periaortic application of $\mathrm{CaCl}_{2}$ as previously described (19). Sham group animals $(n=10)$ were treated with saline instead of $\mathrm{CaCl}_{2}$. Following surgery, the $\mathrm{CaCl}_{2}$-induced TAA rats were then randomly divided into the curcumin and vehicle groups. The curcumin group $(n=10)$ was treated daily by oral gavage with $100 \mathrm{mg} / \mathrm{kg}$ curcumin (Sigma-Aldrich; Merck KGaA, Darmstadt, Germany) suspended in $1 \%$ carboxymethyl cellulose. The vehicle group $(n=10)$ and sham group were treated daily by oral gavage with the same volume of $1 \%$ carboxymethyl cellulose only. The dose of curcumin was based on our previous experiment (19). Following 28 days, the rats were humanely sacrificed, and whole hearts and aortas were excised and analyzed.

Tissue collection and processing. The samples were either frozen in liquid nitrogen or fixed at $4^{\circ} \mathrm{C}$ in $4 \%$ phosphate-buffered formalin for $24 \mathrm{~h}$. For each subject, one part of the aneurysm was used for histological examination and the other part was used for western blot analysis.

Aortic diameter measurements of rat aortas. To accurately measure the aortic diameter, the aneurysm diameters were calculated using light microscopy (Olympus SZH Stereo Zoom microscope; Olympus Corporation, Tokyo, Japan) as previously described (19). Digital images of the descending thoracic aorta were obtained using a color charge-coupled device camera (7.2 Color Mosaic; Micro Video Instruments, Inc., Avon, MA, USA) linked to a computer with digital imaging software (SPOT Advanced version 3.5; Micro Video Instruments, Inc.).

Histological analysis. Aortas were fixed at $4{ }^{\circ} \mathrm{C}$ in $4 \%$ PBS-buffered paraformaldehyde for $24 \mathrm{~h}$ and then embedded in paraffin. For histological analysis, aortic tissue cross-sections $(5 \mu \mathrm{m})$ from the human patients and rats were prepared. Sections were deparaffinized at room temperature with 2 applications of xylene for $10 \mathrm{~min}$ each. They were then rehydrated with 2 applications of absolute alcohol for $5 \mathrm{~min}$ each, and placed in distilled water. Hematoxylin and eosin (H\&E) was applied to analyze general tissue morphology. Hematoxylin staining solution was added for $8 \mathrm{~min}$ at room temperature, followed by $1 \%$ acid alcohol for $30 \mathrm{sec}$, then tissues were counterstained with eosin-phloxine solution for $1 \mathrm{~min}$ at room temperature. Sections were then dehydrated with absolute alcohol, cleared with xylene and mounted. The change in elastic lamellar was observed by Orcein staining. The prepared sections placed in distilled water were stained in $1 \%$ Orcein for $1 \mathrm{~h}$ at room temperature, then dehydrated with 2 applications ( 5 min each) of absolute alcohol, cleared with 2 applications (10 min each) of xylene and mounted. For immunohistochemical analysis, aortic cross sections were deparaffinized twice in xylene (10 min each), rehydrated twice with absolute alcohol (5 min each) and placed in distilled water. Following heat-induced antigen retrieval in a microwave, the cross sections were blocked at room temperature with $3 \%$ hydrogen peroxide for $10 \mathrm{~min}$. Diluted primary antibodies were applied to the sections and incubated overnight at $4^{\circ} \mathrm{C}$. The primary antibodies, anti-VEGF (cat. no. bs-1313R; 1:150; BIOSS, Beijing, China), anti-CD3 (cat. no. MCA772; 1:200; T cell marker; Bio-Rad Laboratories, Inc., Hercules, CA, USA) and anti-CD68 (cat. no. BA3638; 1:200; macrophage marker; Wuhan Boster Biological Technology, Ltd., Wuhan, China) were used to locate inflammatory cell infiltration in the aortic walls. Immune complexes were visualized using 3,3'-diaminobenzidine staining and slides were counterstained with hematoxylin. All sections were then dehydrated in graded alcohols and covered by coverslips with neutral balsam for light microscopy. For negative control experiments, the primary antibodies were omitted and the sections were processed as aforementioned. Non-specific staining was not observed. For all sections, the inflammatory cells were determined by counting 

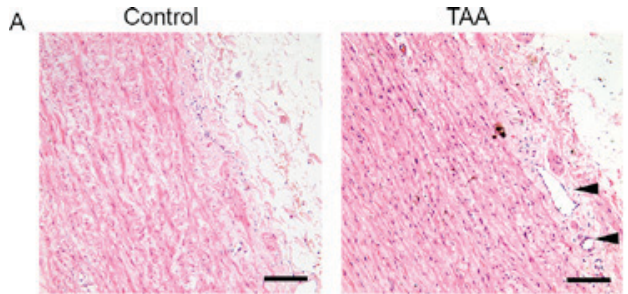

C

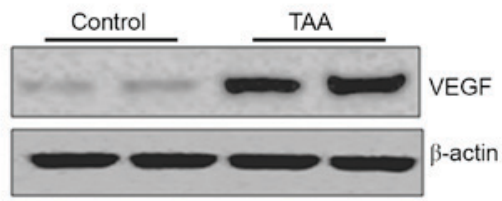

B
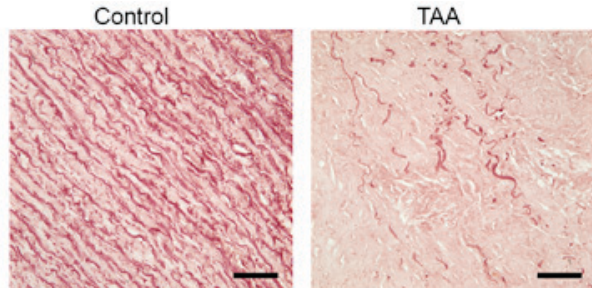

$\mathrm{D}$

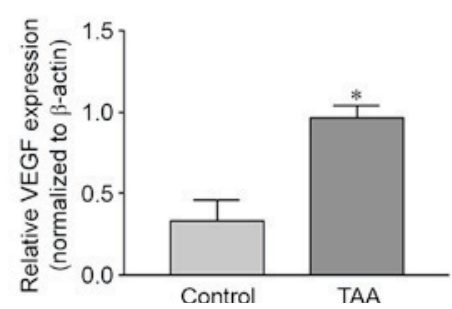

Figure 1. Histological alterations and VEGF expression in human TAA samples. (A) Hematoxylin and eosin staining revealed the microvessels in human TAA samples. Arrows indicate microvessels. (B) Orcein staining was used to detect the structure of elastin in human TAA samples. (C) Representative images of immunoblots for VEGF. Results were normalized to $\beta$-actin. (D) Semi-quantitative analysis of VEGF expression in human TAA samples. All values are presented as the mean \pm standard error of the mean ( $\mathrm{n}=4$ samples). ${ }^{*} \mathrm{P}<0.05$ vs. control group. Scale bars $=100 \mu \mathrm{m}$. TAA, thoracic aortic aneurysm; VEGF, vascular endothelial growth factor.

the mean number of nuclei surrounded by positive immunostaining within $\mathrm{x} 400$ high power fields (5-6 fields of view/section).

Western blot analysis. Frozen aortic tissues from patients, and aortic tissues and whole hearts from rats, were lysed in radioimmunoprecipitation buffer containing a protease inhibitor cocktail, $1 \mathrm{mM} \beta$-glycerolphosphate, $2 \mathrm{mM}$ sodium vanadate, $2.5 \mathrm{mM}$ sodium pyrophosphate, $1 \mathrm{mM}$ EDTA and $1 \mathrm{mM}$ EGTA. Total protein levels were measured using a bicinchoninic acid protein assay kit (Beyotime Institute of Biotechnology, Shanghai, China). Proteins (40 $\mu \mathrm{g})$ were then separated by $10 \%$ SDS-PAGE and were transferred onto polyvinylidene fluoride membranes (EMD Millipore, Billerica, MA, USA). The membranes were blocked with $5 \%$ skim milk in TBS containing $0.1 \%$ Tween-20 for $2 \mathrm{~h}$ at room temperature and were then incubated with primary antibodies overnight at $4^{\circ} \mathrm{C}$. The following primary antibodies were used: Anti-VEGF (cat. no. bs-1313R; 1:500; BIOSS), anti-monocyte chemoattractant protein-1 (cat. no. bs-1101R; MCP-1; 1:1,000; BIOSS), anti-tumor necrosis factor (TNF)- $\alpha$ (cat. no. bs-2081Rl; 1:1,000; BIOSS), anti-vascular cell adhesion molecule-1 (cat. no. sc-13160; VCAM-1; 1:1,000; Santa Cruz Biotechnology, Inc., Dallas, TX, USA), anti-intercellular adhesion molecule-1 (cat. no. sc-8439; ICAM-1; 1:1,000; Santa Cruz Biotechnology, Inc.) and rabbit anti- $\beta$-actin (cat. no. bs-0061R; 1:1,000; BIOSS). The membranes were then incubated with horseradish peroxidase (HRP)-conjugated goat anti-rabbit IgG (cat. no. bs-0295G; 1:5,000; BIOSS) and HRP-conjugated goat anti-mouse IgG (cat. no. bs-0296G; 1:5,000; BIOSS) for $1 \mathrm{~h}$ at room temperature. They were visualized using an enhanced chemiluminescence kit (Beyotime Institute of Biotechnology) and ChemDoc XRS with Quantity One version 4.1 software (Bio-Rad Laboratories, Inc.). The results were normalized to those of $\beta$-actin. Blots were repeated at least three times for each condition. To quantify and compare the levels of proteins, the density of each band was measured by densitometry (Image Lab software version 4.1; Bio-Rad Laboratories, Inc.).
Statistical analysis. Terminal aortic size was expressed as a percentage increase from the baseline size in each rat. The mean percentage of change was then determined and compared to the control group. All values are expressed as the mean \pm standard error of the mean. All statistical analyses were carried out using IBM SPSS Statistics (version 19; IBM Corp., Armonk, NY, USA) and experiments were repeated three times. One-way analysis of variance with the Dunnett's post hoc test were used to determine the significance of differences in multiple comparisons. $\mathrm{P}<0.05$ was considered to indicate a statistically significant difference.

\section{Results}

Histological analysis of the human TAA samples. H\&E and Orcein staining were performed in human TAA samples. H\&E staining revealed that there was an increase in microvessel density in the medial and adventitia of the human TAA samples when compared with the control aortic samples. In addition, Orcein staining identified degradation in the wavy structure of elastin when compared with the control aortic samples (Fig. 1A and B).

VEGF expression in human TAA. It has recently been reported that VEGF has an important role in the pathogenesis of AAA (12). Therefore, the present study detected the expression levels of VEGF in TAA. Western blotting demonstrated that the expression of VEGF was significantly increased in TAA samples compared with the control aortic samples (Fig. 1C and D).

Aortic diameter in the rat TAA model. To examine the effects of curcumin on vasculogenesis and inflammation in TAA, a rat model of TAA was established, and rats were then treated with curcumin daily for 4 weeks. Aortic diameter was measured in the TAA animal model 28 days following the operation. The diameter of the thoracic aorta was larger in the vehicle group compared with in the sham-operated group. However, 
A

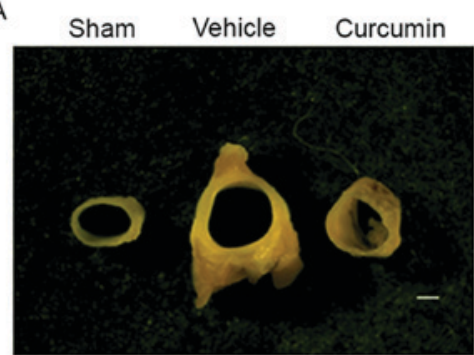

B

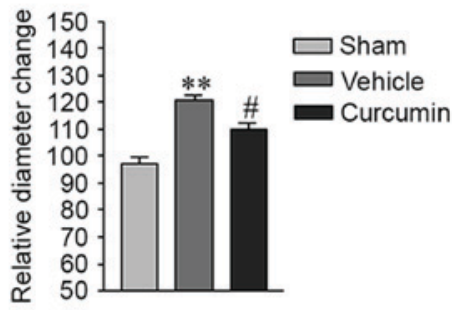

C

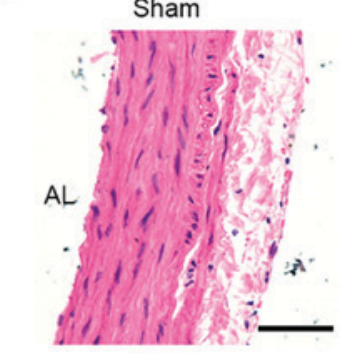

D

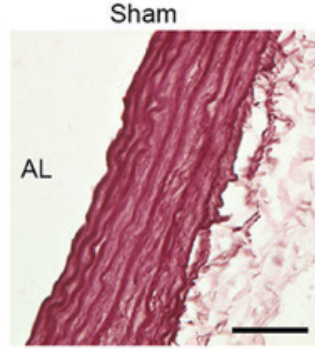

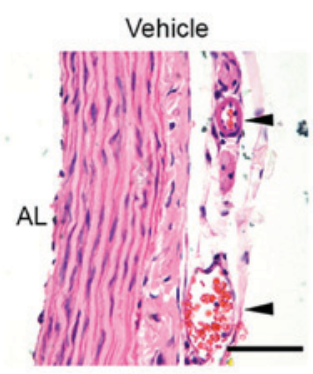

Vehicle

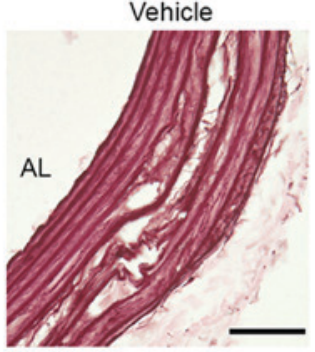

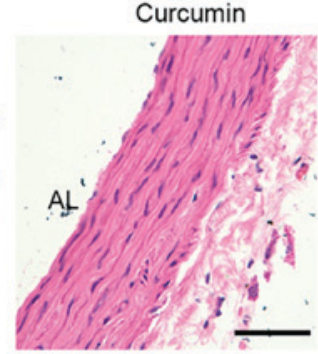

Curcumin

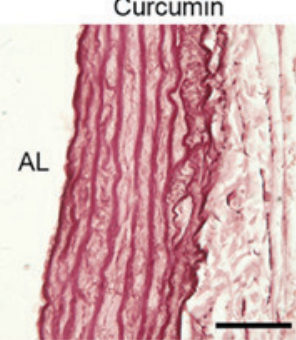

Figure 2. Curcumin prevents aortic dilation in the rat TAA model. (A) Representative macroscopic appearances of the aorta in the different groups (B) Relative diameter change in the thoracic aorta following the operation. (C) Representative hematoxylin and eosin-stained sections showing microvessels in the aortic wall. Arrows indicate microvessels. (D) Representative Orcein-stained sections showing alterations in the elastic lamellar. Data are presented as the mean \pm standard error of the mean. ${ }^{* *} \mathrm{P}<0.01$ vs. sham control group; ${ }^{*} \mathrm{P}<0.05$ vs. vehicle group. Scale bars $=1,000 \mu \mathrm{m}$ in $(\mathrm{A})$, and $50 \mu \mathrm{m}$ in $(\mathrm{C}$ and $\mathrm{D})$. TAA, thoracic aortic aneurysm; AL, aortic lumen.

A

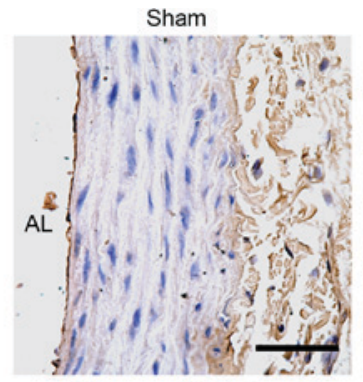

B
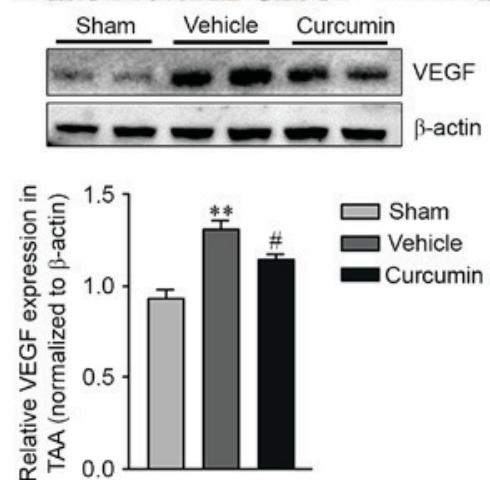

Vehicle

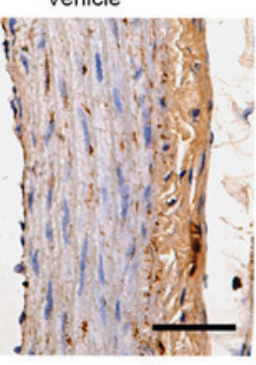

C
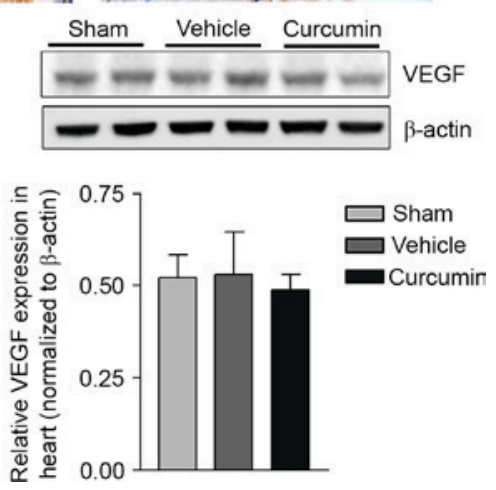

Figure 3. Curcumin reduces the expression of VEGF in rat TAA. (A) Immunohistochemical staining identified the location of VEGF in TAA. Brown deposits indicate positive staining. Scale bars $=50 \mu \mathrm{m}$. (B) Representative images of immunoblots are shown for VEGF expression in TAA. $\beta$-actin was used as an internal control. (C) Representative images of immunoblots are shown for VEGF expression in the heart. $\beta$-actin was used as an internal control. All values are presented as the mean \pm standard error of the mean $\left(\mathrm{n}=4\right.$ samples). ${ }^{* *} \mathrm{P}<0.01$ vs. sham control group; ${ }^{\#} \mathrm{P}<0.05$ vs. vehicle group. TAA, thoracic aortic aneurysm; VEGF, vascular endothelial growth factor; AL, aortic lumen. 

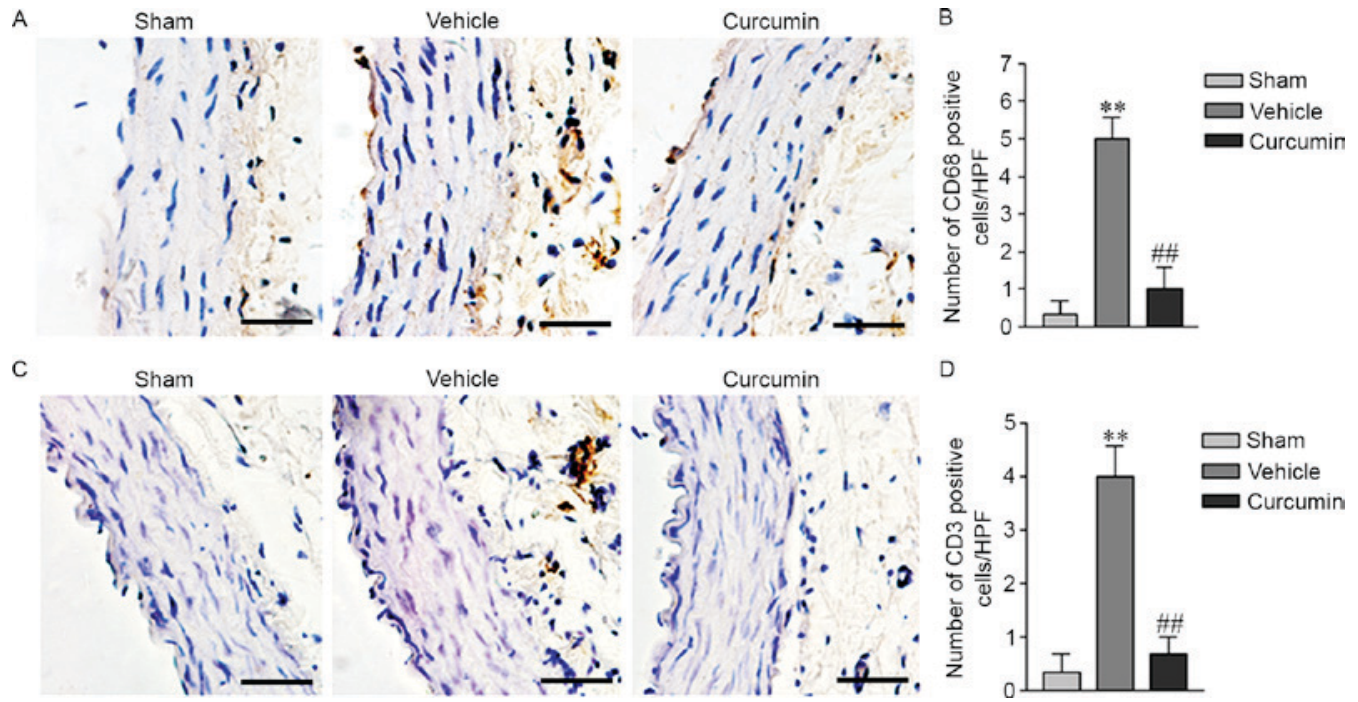

D

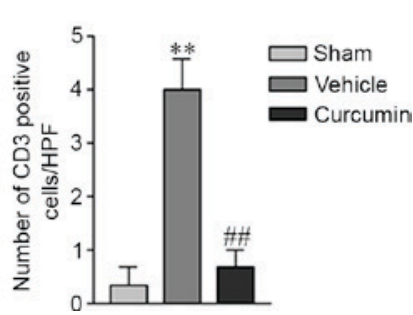

Figure 4. Curcumin prevents the infiltration of macrophages and T cells in rat TAA. (A) Representative photomicrographs of CD68 (macrophages) immunostaining in aortic sections. Brown staining indicates positive cells. (B) Semi-quantitative analysis of infiltration of CD68 ${ }^{+}$cells in aortic sections (C) Representative photomicrographs of CD3 (T cells) immunostaining in aortic sections. Brown staining indicates positive cells. (D) Semi-quantitative analysis of infiltration of $\mathrm{CD}^{+}$cells in aortic sections. Scale bars $=50 \mu \mathrm{m}$. Data are presented as the mean \pm standard error of the mean ( $\mathrm{n}=4 \mathrm{samples)}$. ${ }^{* *} \mathrm{P}<0.01$ vs. sham control group; ${ }^{\# \#} \mathrm{P}<0.01$ vs. vehicle group. TAA, thoracic aortic aneurysm; $\mathrm{CD}$, cluster of differentiation.

A

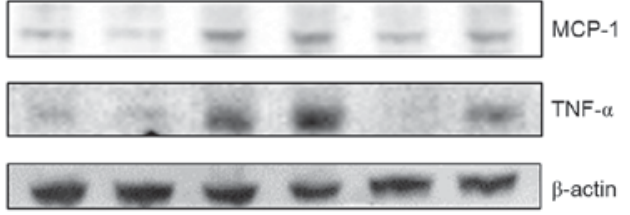

B

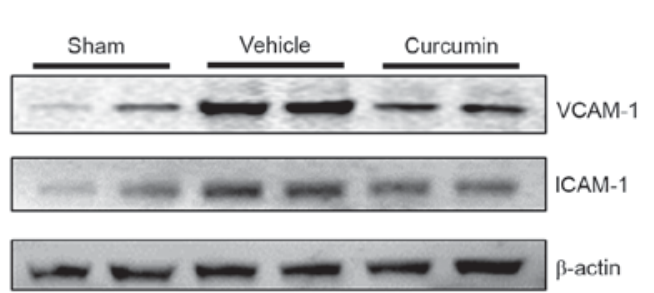

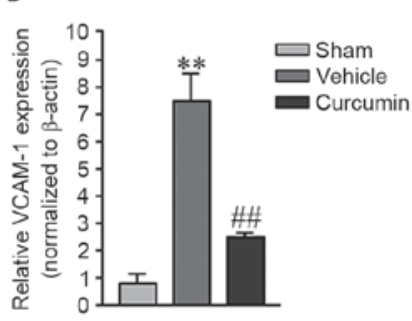

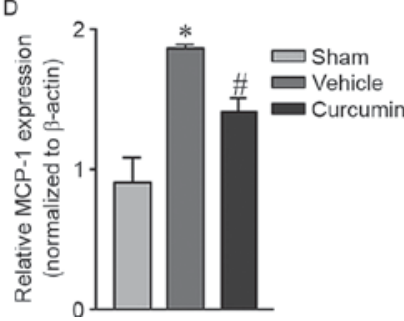

C
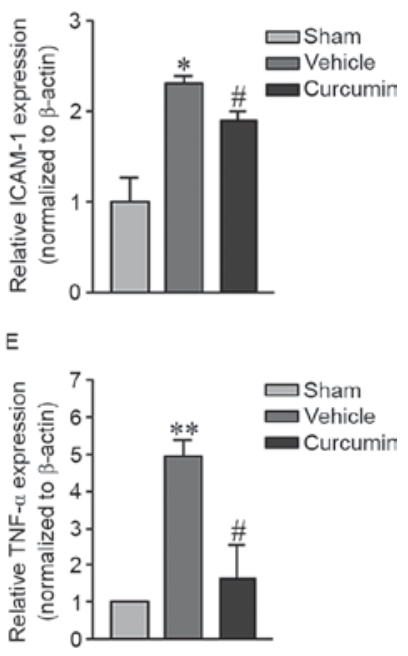

Figure 5. Curcumin decreases the expression of inflammatory factors in rat TAA. (A) Representative western blotting of VCAM-1, ICAM-1, MCP-1 and TNF- $\alpha$ expression. Results were normalized to $\beta$-actin. Semi-quantitative analysis of (B) VCAM-1, (C) ICAM-1, (D) MCP-1 and (E) TNF- $\alpha$. "P<0.05 and ${ }^{* *} \mathrm{P}<0.01$ vs. sham control group; ${ }^{\#} \mathrm{P}<0.05$ and ${ }^{\# \#} \mathrm{P}<0.01$ vs. vehicle group. TAA, thoracic aortic aneurysm; VCAM-1, vascular cell adhesion molecule-1; ICAM-1, intercellular adhesion molecule-1; MCP-1, monocyte chemoattractant protein-1; TNF- $\alpha$, tumor necrosis factor- $\alpha$.

treatment with curcumin resulted in a decrease in thoracic aortic diameter compared with in the vehicle group (Fig. 2A and $\mathrm{B}$ ). These results revealed that TAA may be prevented in $\mathrm{CaCl}_{2}$-treated rats by curcumin treatment.

Histological analysis in the rat TAA model. As observed in the human TAA samples, H\&E staining revealed that the number of microvessels in the aortic wall was increased in the adventitia of the rat TAA samples compared with the control group. However, this effect was attenuated by treatment with curcumin (Fig. 2C).

In parallel with thoracic aortic dilatation with $\mathrm{CaCl}_{2}$ treatment, vascular elastin integrity was altered, as determined by Orcein staining (Fig. 2D). The aortic wall of the sham group was intact without damage. However, in the TAA vehicle group, the medial elastic lamellae in some parts of the aorta lost its wave shape and developed fragmentations. Conversely, administration of curcumin inhibited elastin fragmentation and preserved the elastic laminar wave (Fig. 2D). These results suggested that curcumin treatment may effectively decrease the formation of microvessels and elastin degeneration in the rat TAA model.

Curcumin decreases the expression of VEGF in the rat TAA model. To determine the effects of curcumin on the expression levels of VEGF in TAA, immunohistochemical staining and western blotting were performed. There was a low expression of VEGF in the normal aorta; however, in vehicle-treated rats 
the expression of VEGF in the aorta was significantly increased when compared with the sham group. In addition, curcumin treatment significantly reduced the expression of VEGF when compared with the vehicle group (Fig. 3A and B). To further determine whether curcumin had an inhibitory effect on other organs, the rat heart tissues were homogenized and total proteins extracted. Western blot analysis revealed that VEGF expression was not decreased in the curcumin-treated group, which indicated that curcumin treatment may not affect VEGF expression in other organs (Fig. 3C).

Curcumin decreases inflammatory cell infiltration in TAA. To evaluate inflammatory cell infiltration in the vascular wall, immunostaining of CD68 (macrophage marker) and CD3 ( $\mathrm{T}$ cell marker) was conducted, and the number of positive cells was counted. The number of $\mathrm{CD}^{+} 8^{+}$(Fig. 4A and B) and $\mathrm{CD}^{+}$(Fig. 4C and D) cells was increased in the aortas of TAA rats compared with the sham rats, indicating that there may be increased infiltration of macrophages and T cells in TAA. However, treatment with curcumin inhibited macrophage and $\mathrm{T}$ cell infiltration in the aortas of TAA rats when compared with the vehicle group.

Curcumin decreases the expression of inflammatory factors in TAA. VCAM-1, ICAM-1 and TNF- $\alpha$ are markers of immune-inflammatory activation. Curcumin decreased inflammatory cell infiltration in the aorta; therefore, the associated inflammatory factors were assessed by western blotting (Fig. 5A). The protein expression levels of VCAM-1 (Fig. 5B), ICAM-1 (Fig. 5C), MCP-1 (Fig. 5D) and TNF- $\alpha$ (Fig. 5E) were significantly increased in the aortic walls of vehicle-treated rats compared with sham-operated rats. Conversely, curcumin significantly decreased the levels of inflammatory factors in the vehicle-treated rats, to a comparable level to the sham control group. These results indicated that curcumin effectively decreased inflammatory activation in the aorta in TAA.

\section{Discussion}

The present study demonstrated that angiogenesis occurred in human TAA, and VEGF expression was increased in TAA samples compared with healthy aortas. Administration of curcumin significantly suppressed the development of rat TAA. Reductions in vascular pathologies during curcumin administration were associated with decreased neovascularization and VEGF expression in TAA. In addition, curcumin treatment decreased inflammatory cell infiltration and the expression of associated inflammatory factors in TAA.

Angiogenesis is associated with the progression of aortic aneurysm, and treatment with an angiogenesis inhibitor may attenuate AAA formation $(12,24)$. VEGF is a potent angiogenic factor and vascular permeability-enhancing cytokine that is overexpressed during tissue repair processes and chronic inflammation (25). The presence of VEGF-expressing cells in the vessel wall is strongly associated with the degree of neovascularization and aortic aneurysm rupture (26). In vitro administration of VEGF to human smooth muscle cells has been reported to significantly enhance the production of active matrix metalloproteinases, which serve an important pathogenic role in vascular damage in aortic aneurysms by promoting matrix degradation and angiogenesis (27). The present study demonstrated that VEGF levels were significantly increased in patients with aortic aneurysm when compared with controls, which is consistent with the results of a previous study (12). Therefore, it may be hypothesized that VEGF is highly expressed in aneurysm tissue and suppression of VEGF may prevent the progression of TAA.

Kaneko et al (12) revealed that inhibition of VEGF is an important event in suppressing aneurysm progression, and in the regression of aneurysms. Curcumin has previously been studied for its wide-range of effects on tumorigenesis, angiogenesis, apoptosis and signal transduction pathways (28). In addition, curcumin has been demonstrated to be safe in human trials (29). It has also been revealed that curcumin may interfere with VEGF gene expression and inhibit the expression of the receptor, VEGF receptor type-2, in human umbilical vein endothelial cells (30). Therefore, it is reasonable to speculate that inhibition of VEGF by curcumin may prevent or effectively slow the progression of TAA. As expected, the present study demonstrated an inhibitory effect of curcumin on the progression of TAA, and curcumin treatment significantly inhibited vasculogenesis and VEGF expression in TAA.

Angiogenesis during postnatal development is usually associated with inflammation (31). Previous studies have highlighted the importance of inflammation in the pathogenesis of aortic aneurysm $(32,33)$. A previous study demonstrated that in human TAA, there is a significant increase in the number of $\mathrm{T}$ lymphocytes and macrophages compared with in control aortas (34). In the present study, infiltration of $\mathrm{T}$ cells and macrophages was detected in the rat model of TAA; however, curcumin significantly decreased inflammatory cell infiltration in TAA. MCP-1-mediated macrophage recruitment and macrophage-induced inflammation are critical for aortic aneurysm formation and aortic dissection (35-37). In addition, inhibiting MCP-1 signaling via various methods may markedly suppress AAA development $(38,39)$. The present study detected MCP-1 expression and revealed that MCP-1 expression was significantly increased in TAA. This increase was markedly attenuated by curcumin treatment. The adhesion molecules VCAM-1 and ICAM-1, which are associated with leukocyte attachment, rolling and transendothelial migration, serve a significant role in the onset of vascular inflammation (40). Aortic aneurysm is also associated with increased levels of TNF- $\alpha$, which mediates the inflammatory response, downregulates elastin gene expression and initiates aneurysm formation $(41,42)$, in addition to VCAM-1 and ICAM-1, suggesting an important role for immunological factors in aortic aneurysm progression. In the present study, TNF- $\alpha$, VCAM-1 and ICAM-1, as markers of immune-inflammatory activation, were significantly increased in the aorta of TAA rats compared with in sham-operated rats. However, curcumin markedly decreased these inflammatory factors in TAA.

In conclusion, to the best of our knowledge, the present study is the first to indicate that oral administration of curcumin can suppress the development of TAA via mechanisms associated with the inhibition of angiogenesis and inflammation. Based on the percentage increase in aortic diameter in the rat model, with respect to what is known in clinical patients, curcumin treatment may prevent the requirement for surgery in patients with an aorta $>6 \mathrm{~cm}$ in diameter. 


\section{Acknowledgements}

The present study was supported by the National Natural Science Foundation of China under Contract (grant no. 81571919).

\section{References}

1. Cormack SM and Owens WA: Aneurysmal dilation of the ascending thoracic aorta and the aortic arch following surgical repair of type A dissection. BMJ Case Rep 2012: pii: bcr2012006963, 2012

2. Guo DC, Papke CL, He R and Milewicz DM: Pathogenesis of thoracic and abdominal aortic aneurysms. Ann N Y Acad Sci 1085: 339-352, 2006.

3. Escudero P, Navarro A,Ferrando C,FurioE, Gonzalez-Navarro H, Juez M, Sanz MJ and Piqueras L: Combined treatment with bexarotene and rosuvastatin reduces angiotensin-II-induced abdominal aortic aneurysm in apoE(-/-) mice and angiogenesis. Br J Pharmacol 172: 2946-2960, 2015.

4. Forsythe RO, Newby DE and Robson JM: Monitoring the biological activity of abdominal aortic aneurysms beyond ultrasound. Heart 102: 817-824, 2016

5. Choke E, Thompson MM, Dawson J, Wilson WR, Sayed S, Loftus IM and Cockerill GW: Abdominal aortic aneurysm rupture is associated with increased medial neovascularization and overexpression of proangiogenic cytokines. Arterioscler Thromb Vasc Biol 26: 2077-2082, 2006.

6. Kessler K, Borges LF, Ho-Tin-Noé B, Jondeau G, Michel JB and Vranckx R: Angiogenesis and remodelling in human thoracic aortic aneurysms. Cardiovasc Res 104: 147-159, 2014.

7. Nesi G, Anichini C, Tozzini S, Boddi V, Calamai G and Gori F: Pathology of the thoracic aorta: A morphologic review of 338 surgical specimens over a 7-year period. Cardiovasc Pathol 18: 134-139, 2009.

8. Johnston WF, Salmon M, Pope NH, Meher A, Su G, Stone ML, Lu G, Owens GK, Upchurch GR Jr and Ailawadi G: Inhibition of interleukin- $1 \beta$ decreases aneurysm formation and progression in a novel model of thoracic aortic aneurysms. Circulation 130 (11 Suppl 1): S51-S59, 2014.

9. Agaimy A, Weyand $\mathrm{M}$ and Strecker T: Inflammatory thoracic aortic aneurysm (lymphoplasmacytic thoracic aortitis): A 13-year-experience at a German Heart Center with emphasis on possible role of IgG4. Int J Clin Exp Pathol 6: 1713-1722, 2013.

10. Nishibe T, Dardik A, Kondo Y, Kudo F, Muto A, Nishi M, Nishibe M and Shigematsu H: Expression and localization of vascular endothelial growth factor in normal abdominal aorta and abdominal aortic aneurysm. Int Angiol 29: 260-265, 2010.

11. Senger DR, Galli SJ, Dvorak AM, Perruzzi CA, Harvey VS and Dvorak HF: Tumor cells secrete a vascular permeability factor that promotes accumulation of ascites fluid. Science 219 : 983-985, 1983.

12. Kaneko H, Anzai T, Takahashi T, Kohno T, Shimoda M, Sasaki A, Shimizu H, Nagai T, Maekawa Y, Yoshimura K, et al: Role of vascular endothelial growth factor-A in development of abdominal aortic aneurysm. Cardiovasc Res 91: 358-367, 2011.

13. Mirzaei H, Shakeri A, Rashidi B, Jalili A, Banikazemi Z and Sahebkar A: Phytosomal curcumin: A review of pharmacokinetic, experimental and clinical studies. Biomed Pharmacother 85: $102-112,2017$.

14. Goozee KG, Shah TM, Sohrabi HR, Rainey-Smith SR, Brown B, Verdile G and Martins RN: Examining the potential clinical value of curcumin in the prevention and diagnosis of Alzheimer's disease. Br J Nutr 115: 449-465, 2016

15. Jiang S, Han J, Li T, Xin Z, Ma Z, Di W, Hu W, Gong B, Di S, Wang D and Yang Y: Curcumin as a potential protective compound against cardiac diseases. Pharmacol Res 119: 373-383, 2017.

16. Huang R, Liu Y, Xiong Y, Wu H, Wang G, Sun Z, Chen J, Yan X, Pan Z, Xia J, et al: Curcumin protects against liver fibrosis by attenuating infiltration of Grlhi monocytes through inhibition of monocyte chemoattractant protein-1. Discov Med 21: 447-457, 2016.

17. Allegra A, Innao V, Russo S, Gerace D, Alonci A and Musolino C: Anticancer Activity of curcumin and its analogues: Preclinical and clinical studies. Cancer Invest 35: 1-224, 2017.
18. Parodi FE, Mao D, Ennis TL, Pagano MB and Thompson RW: Oral administration of diferuloylmethane (curcumin) suppresses proinflammatory cytokines and destructive connective tissue remodeling in experimental abdominal aortic aneurysms. Ann Vasc Surg 20: 360-368, 2006.

19. Fan J, Li X, Yan YW, Tian XH, Hou WJ, Tong H and Bai SL: Curcumin attenuates rat thoracic aortic aneurysm formation by inhibition of the c-Jun N-terminal kinase pathway and apoptosis. Nutrition 28: 1068-1074, 2012.

20. Arbiser JL, Klauber N, Rohan R, van Leeuwen R, Huang MT, Fisher C, Flynn E and Byers HR: Curcumin is an in vivo inhibitor of angiogenesis. Mol Med 4: 376-383, 1998.

21. Tung YT, Chen HL, Lai CW, Shen CJ, Lai YW and Chen CM: Curcumin reduces pulmonary tumorigenesis in vascular endothelial growth factor (VEGF)-overexpressing transgenic mice. Mol Nutr Food Res 55: 1036-1043, 2011.

22. Binion DG, Otterson MF and Rafiee P: Curcumin inhibits VEGF-mediated angiogenesis in human intestinal microvascular endothelial cells through COX-2 and MAPK inhibition. Gut 57: 1509-1517, 2008

23. National Research Council (US) Institute for Laboratory Animal Research: Guide for the Care and Use of Laboratory Animals. Washington (DC): National Academies Press (US), 1996.

24. Kaneko H, Anzai T, Morisawa M, Kohno T, Nagai T, Anzai A, Takahashi T, Shimoda M,Sasaki A, Maekawa Y, et al: Resveratrol prevents the development of abdominal aortic aneurysm through attenuation of inflammation, oxidative stress, and neovascularization. Atherosclerosis 217: 350-357, 2011.

25. Alkim C, Sakiz D, Alkim H, Livaoglu A, Kendir T, Demirsoy H, Erdem L, Akbayir N and Sokmen M: Thrombospondin-1 and VEGF in inflammatory bowel disease. Libyan J Med: 7, 2012.

26. PalmieriD,Pane B, Barisione C, Spinella G, Garibaldi S, GhigliottiG, Brunelli C, Fulcheri E and Palombo D: Resveratrol counteracts systemic and local inflammation involved in early abdominal aortic aneurysm development. J Surg Res 171: e237-e246, 2011.

27. Wang H and Keiser JA: Vascular endothelial growth factor upregulates the expression of matrix metalloproteinases in vascular smooth muscle cells: Role of flt-1. Circ Res 83: 832-840, 1998.

28. Mrudula T, Suryanarayana P, Srinivas PN and Reddy GB: Effect of curcumin on hyperglycemia-induced vascular endothelial growth factor expression in streptozotocin-induced diabetic rat retina. Biochem Biophys Res Commun 361: 528-532, 2007.

29. Chainani-Wu N: Safety and anti-inflammatory activity of curcumin: A component of tumeric (Curcuma longa). J Altern Complement Med 9: 161-168, 2003.

30. El-Azab M, Hishe H, Moustafa Y and El-Awady el-S: Anti-angiogenic effect of resveratrol or curcumin in Ehrlich ascites carcinoma-bearing mice. Eur J Pharmacol 652: 7-14, 2011.

31. Szade A, Grochot-Przeczek A, Florczyk U, Jozkowicz A and Dulak J: Cellular and molecular mechanisms of inflammation-induced angiogenesis. IUBMB Life 67: 145-159, 2015.

32. Jalalzadeh $H$, Indrakusuma $R$, Planken $R N$, Legemate $D A$, Koelemay MJ and Balm R: Inflammation as a predictor of abdominal aortic aneurysm growth and rupture: A systematic review of imaging biomarkers. Eur J Vasc Endovasc Surg 52: 333-42, 2016.

33. Potteaux S and Tedgui A: Monocytes, Macrophages and Other Inflammatory Mediators of Abdominal Aortic Aneurysm. Curr Pharm Des 21: 4007-4015, 2015.

34. He R, Guo DC, Sun W, Papke CL, Duraisamy S, Estrera AL, Safi HJ, Ahn C, Buja LM, Arnett FC, et al: Characterization of the inflammatory cells in ascending thoracic aortic aneurysms in patients with Marfan syndrome, familial thoracic aortic aneurysms, and sporadic aneurysms. J Thorac Cardiovasc Surg 136: 922-929, 2008.

35. Spin JM, Hsu M, Azuma J, Tedesco MM, Deng A, Dyer JS, Maegdefessel L, Dalman RL and Tsao PS: Transcriptional profiling and network analysis of the murine angiotensin II-induced abdominal aortic aneurysm. Physiol Genomics 43: 993-1003, 2011.

36. Colonnello JS, Hance KA, Shames ML, Wyble CW, Ziporin SJ, Leidenfrost JE, Ennis TL, Upchurch GR Jr and Thompson RW: Transient exposure to elastase induces mouse aortic wall smooth muscle cell production of MCP-1 and RANTES during development of experimental aortic aneurysm. J Vasc Surg 38: 138-146, 2003.

37. Tieu BC, Lee C, Sun H, Lejeune W, Recinos A III, Ju X, Spratt H, Guo DC, Milewicz D, Tilton RG and Brasier AR: An adventitial IL-6/MCP1 amplification loop accelerates macrophage-mediated vascular inflammation leading to aortic dissection in mice. J Clin Invest 119: 3637-3651, 2009. 
38. de Waard V, Bot I, de Jager SC, Talib S, Egashira K, de Vries MR, Quax PH, Biessen EA and van Berkel TJ: Systemic MCP1/CCR2 blockade and leukocyte specific MCP1/CCR2 inhibition affect aortic aneurysm formation differently. Atherosclerosis 211: 84-89, 2010.

39. Aoki T, Yamamoto K, Fukuda M, Shimogonya Y, Fukuda S and Narumiya S: Sustained expression of MCP-1 by low wall shear stress loading concomitant with turbulent flow on endothelial cells of intracranial aneurysm. Acta Neuropathol Commun 4: 48, 2016.

40. Fan J, Li X, Zhong L, Hao-Tong, Di J, Liu F, Zhao HH and Bai SL: MCP-1, ICAM-1 and VCAM-1 are present in early aneurysmal dilatation in experimental rats. Folia Histochem Cytobiol 48: 455-461, 2010.
41. Jayaraman T, Paget A, Shin YS, Li X, Mayer J, Chaudhry H, Niimi Y, Silane M and Berenstein A: TNF-alpha-mediated inflammation in cerebral aneurysms: A potential link to growth and rupture. Vasc Health Risk Manag 4: 805-817, 2008.

42. Yan YW, Fan J, Bai SL, Hou WJ, Li X and Tong H: Zinc Prevents abdominal aortic aneurysm formation by induction of A20-mediated suppression of NF- $\mathrm{B}$ Pathway. PLoS One 11: e0148536, 2016. 